Mon. Not. R. Astron. Soc. 000, 1 (2002) Printed 20 December $2018 \quad$ (MN LATEX style file v2.2)

\title{
Non-Gaussian Tails of Cosmological Density Distribution Function from Dark Halo Approach
}

\author{
Atsushi Taruya ${ }^{1}$, Takashi Hamana ${ }^{2}$ and Issha Kayo ${ }^{3}$ \\ ${ }^{1}$ Research Center for the Early Universe(RESCEU), School of Science, University of Tokyo, Tokyo 113-0033, Japan \\ 2 National Astronomical Observatory, Mitaka, Tokyo 181-8588, Japan \\ 3 Department of Physics, University of Tokyo, Tokyo 113-0033, Japan
}

20 December 2018

\begin{abstract}
We present a simple model based on the dark halo approach which provides a useful way to understand key points determining the shape of the non-Gaussian tails of the dark matter one-point probability distribution function(PDF). In particular, using the scale-free models with power-law profile of dark halos, we derive a simple analytic expression for the one-point PDF. It is found that the shape of the PDF changes at the characteristic value of $\delta_{*}$ which is defined by the smoothed density of a halo with the characteristic mass $M_{*}$ at the epoch. In cold dark matter models with top-hat smoothing filters, the characteristic smoothed density at present time typically takes the value $\delta_{*} \gg 1$ for a small smoothing scale $R_{\mathrm{th}} \sim 1 \mathrm{Mpc} / h$ and conversely $\delta_{*} \ll 1$ for a large smoothing scale $R_{\mathrm{th}}>10 \mathrm{Mpc} / h$. On the range $\delta / \delta_{*}<1$, the shape of the PDF is almost solely determined by the outer slope of halos and scales as a power-law. The resultant non-Gaussian tails of PDF then resemble the log-normal PDFs in that range and show a good agreement with N-body simulations, which can be ascribed to the universality of the outer slope of the halo profile. In contrast, tails of one-point $\mathrm{PDF}$ in the range $\delta / \delta_{*}>1$ basically follow the steep exponential tails of the halo mass function, which exhibit a strong sensitivity to both the outer slope of the halo profile and the initial power spectrum. Based on these results, the discussion on the PDF of galaxy distribution and the application to the weak lensing statistics are also presented.
\end{abstract}

Key words: cosmology : theory - dark matter - large-scale structure of Universe

\section{INTRODUCTION}

The probability distribution function (PDF) of the cosmic matter density is one of most fundamental quantities characterizing statistical properties of large-scale structures. In most standard scenarios of the structure formation, the large-scale structures are thought to form by gravitational instabilities from small Gaussian density fluctuations. A deviation in the PDF from the Gaussian distribution is thus most likely caused by the nonlinear gravitational evolution and is a key to understand the structure formation history. The higher order moments of the density field such like the skewness and kurtosis have been used to study the non-Gaussian nature in cosmic density field (e.g., Peebles 1980; Juszkiewicz, Bouchet \& Colombi 1993; Bernardeau 1994a). The one-point PDF contains more statistical information than them.

The galaxy PDFs have been measured from various catalogs (e.g., Hamilton 1985; Bouchet et al. 1993 ; Kofman et al. 1994) and shows significant non-Gaussianity. Indeed it was pointed out that the galaxy PDF is well fitted by the log-normal distribution (e.g., Coles \& Jones 1991; Kofman et al. 1994). The dark matter PDFs measured from $N$-body simulations are also fitted by the log-normal distribution very well (e.g., Bernardeau \& Kofman 1995; Taylor \& Watts 2000). Although the galaxy distribution cannot be regarded as a direct tracer of the dark matter distribution because of unknown biasing relation between them, the similarity in their PDF suggests that there exists a nature common to their distributions.

The origin of the phenomenological log-normal PDF has not yet been clarified. Bernardeau (1994b) and Benardeau \& Kofman (1995) argued that the perturbative construction of one-point PDF shows a strong dependence on the primordial power spectrum and the PDF approaches the log-normal form only when the initial spectrum is proportional to $P(k) \propto k^{n}$ with $n=-1$. On the other hand, using a series of high-resolution N-body simulations, Kayo, Taruya \& Suto (2001) found that the one-point density PDFs with Gaussian initial conditions are approximately described by the log-normal distribution in the strongly non-linear regime. Remarkably, the accuracy of log-normal model turns out to become almost insensitive to the 
underlying power spectrum of density fluctuation, in contrast to the prediction from perturbation theory. While the results of Kayo et al. (2001) show a clear dependence on the primordial initial spectrum in a weakly non-linear regime, consistent with the perturbation theory, there remains crucial to clarify the reason why the PDFs in the simulations tend to become insensitive to the initial power spectrum and approach to the log-normal form in a strongly non-linear regime.

The primary purpose of this paper is to discuss this issue using a simple analytic model. That is, employing the so-called dark halo approach as advocated recently, we construct a model for the non-Gaussian tails of the one-point PDF, which includes the essential ingredient for gravitational clustering and is, at least, consistent with N-body simulations. The basic assumption of our model is that the high density regions after smoothed over with a filter function are mainly attributed to a single massive halo. Clearly, this assumption is violated at the low density regions and we thus restrict our analysis to the highly non-Gaussian tails of mass density PDF. Then, the dark halo approach allows us to derive a simple analytic expression for the one-point PDF in cases with the scale-free initial spectra and power-law profiles of dark halo, from which we explain the qualitative behavior of tails of PDF and its dependences on the halo density profile and on the initial power spectrum. Further, adopting the realistic halo profile and the mass function, the results of our analytic model are quantitatively compared with N-body simulations with various initial power spectra.

In section 2, the basic ingredients for the dark halo approach and an analytic model of tails of one-point PDF are described. Section 3 presents the detailed investigation of the dependence on the halo profile and the sensitivity to the initial power spectra on the non-Gaussian tails of one-point PDF. Assuming the scale-free initial spectrum and the power-law profile of dark halos, we analytically derive an approximate expression for non-Gaussian tails of PDF. Using this result, we attempt to clarify the sensitivity of the one-point PDF to the initial spectra and the halo profiles. In section 4 , the analytic model of one-point PDF is quantitatively compared with those obtained from cosmological N-body simulations. Finally, section $\mathrm{f}$ is devoted to the conclusions and the discussion.

\section{MASS DENSITY PDF FROM THE DARK HALO APPROACH}

\subsection{Basic ingredients}

The basic idea of the dark halo approach which has become very popular recently(e.g., Seljak 2000; Ma \& Fry 2000a; Scoccimarro et al. 2001) is that (i) all the dark matter in the universe belong to one of the virialized clumps (dark halos), and (ii) the matter distribution in the universe is approximated by a sum of the matter distribution within each halo. To construct an analytic model of mass density PDF from the dark halo approach, we need to specify the halo mass function and the density profiles of halos. In the following, we describe these two ingredients.

For the halo mass function, the approximate prescription for counting the number of dark halos has been first presented by Press \& Schechter (1974) and recently an accurate analytic fitting model has been proposed by Sheth \& Tormen (1998). Both models are simply expressed in a unified manner as follows:

$n(M) d M=A\left\{1+\left(\frac{\sigma_{\mathrm{M}}}{\sqrt{a} \delta_{\text {crit }}}\right)^{2 p}\right\} \sqrt{\frac{a}{2 \pi}} \frac{\bar{\rho}}{M} \frac{\delta_{\text {crit }}}{\sigma_{\mathrm{M}}^{3}} \exp \left\{-\frac{a}{2}\left(\frac{\delta_{\text {crit }}}{\sigma_{\mathrm{M}}}\right)^{2}\right\}\left|\frac{d \sigma_{\mathrm{M}}^{2}}{d M}\right| d M$,

where $\bar{\rho}$ is the mean mass density and $\delta_{\text {crit }}$ is the critical over-density of the spherical collapse model(see Nakamura \& Suto 1997 for useful fitting formula). The quantity $\sigma_{\mathrm{M}}$ denotes the linear rms fluctuations of the matter density in a sphere of radius $R_{M}=(3 M / 4 \pi \bar{\rho})^{1 / 3}$. The numerical coefficients $a$ and $p$ are set to $a=1, p=0$ for the Press-Schechter mass function and $a=0.707, p=0.3$ for the mass function by Sheth \& Tormen. The normalization factor $A$ is $\operatorname{determined}$ by $\int n(M) M d M=\bar{\rho}$. Throughout the paper, we extensively use the mass function by Sheth \& Tormen (1998).

The density profile of dark halos is defined by a spherical average for each halo and is given by a function of radius and halo mass. The numerical simulation by Navarro et al. $(1996,1997$; hereafter NFW) revealed that the outer slope of profile becomes -3 , while the inner part asymptotically approaches -1 . In this paper, we adopt their fitting form:

$\frac{\rho_{\mathrm{NFW}}(r ; M)}{\bar{\rho}}=\frac{\delta_{c}}{\left(r / r_{s}\right)\left(1+r / r_{s}\right)^{2}}$

where $\delta_{c}$ and $r_{s}$ respectively denote the characteristic over-density and the scale radius. These parameters are not independent and are characterized in terms of concentration parameter $c \equiv r_{\mathrm{vir}} / r_{s}$, with $r_{\mathrm{vir}}$ being virial radius of the halo. Using this quantity, the characteristic over-density $\delta_{c}$ is given by $\delta_{c}=\left(\Delta_{\mathrm{vir}} / 3\right) c^{3} /[\ln (1+c)-c /(1+c)]$, where $\Delta_{\text {vir }}$ denotes the virial over-density of spherical collapse model (Nakamura \& Suto 1997). For the relation between the concentration parameter and the halo mass, we adopt the following fitting form according to the simulation by Bullock et al. (2001),

$c(M)=c_{*}\left(\frac{M}{M_{*}}\right)^{-\beta}$,

where $M_{*}$ corresponds to the characteristic mass scale satisfying $\sigma_{\mathrm{M}}\left(M_{*}\right)=\delta_{\text {crit }}$. Below in comparing the PDF with the N-body simulation (Sec-4), the parameters $c_{*}$ and $\beta$ are chosen as $\left(c_{*}, \beta\right)=(10,0.15)$ for cold dark matter(CDM) model and $(10,(n+3) / 6)$ for scale-free model with spectral index $n$. The systematic influences of the uncertainty of $c(M)$ on the evaluation of PDF are discussed in detail in Appendix B.

Indeed, recent high-resolution $\mathrm{N}$-body study reveals that the inner slope of halo profile might not converge to -1 , but 
it rather approaches -1.5 (Moore et al. 1999; Fukushige \& Makino 2001a,b; see also Jing \& Suto 2000). Hence, it becomes important to clarify the profile dependence on the non-Gaussian tails of PDF. In this paper, in addition to the NFW profile, we further treat the halo models characterized by a single power-law profile:

$\frac{\rho_{\text {power }}(r ; M)}{\bar{\rho}}=\delta_{c}\left(\frac{r}{r_{s}}\right)^{-\alpha}, \quad(0<\alpha<3)$

which enables us to derive the analytic expressions of PDFs (see Sec.3). Here, the characteristic density and scale radius are determined by the relation between virial mass and virial radius:

$\delta_{c} r_{s}^{\alpha}=\frac{3-\alpha}{3} \Delta_{\mathrm{vir}} r_{\mathrm{vir}}^{\alpha}=\frac{3-\alpha}{3} \Delta_{\mathrm{vir}}\left(\frac{3 M}{4 \pi \bar{\rho} \Delta_{\mathrm{vir}}}\right)^{\alpha / 3}$.

\subsection{Mass density PDFs}

Provided the basic ingredients, we now describe a simple analytic model of mass density PDF.

Let us first describe the cumulative distribution function $P(>\delta ; R)$, which gives a probability that a given density field smoothed over the radius $R$ is larger than $\delta$. Defining the smoothed halo profile:

$\rho_{\text {smooth }}(r ; R, M)=\int d^{3} \boldsymbol{r}^{\prime} W\left(\left|\boldsymbol{r}-\boldsymbol{r}^{\prime}\right| ; R\right) \rho\left(r^{\prime} ; M\right)$,

with the function $W(x ; R)$ being the spherically symmetric smoothing kernel. Since we only consider simple spherical symmetric density distributions of halos, one can calculate the characteristic radius $r(\delta ; R, M)$ by solving the following equation:

$\frac{\rho_{\text {smooth }}[r(\delta ; R, M) ; R, M]}{\bar{\rho}}=\delta+1 \simeq \delta$.

Now we introduce the volume factor $V(>\delta ; R, M)$ which represents, for one halo with mass $M$, the comoving volume in which the smoothed mass density field is always greater than $\delta$. Since the smoothed halo profile is a monotonic decreasing function of the radius, the volume factor is simply given by,

$V(>\delta ; R, M)=\frac{4 \pi}{3}\{r(\delta ; R, M)\}^{3}$.

The volume factor (8) explicitly depends on the halo mass and thereby the volume fraction of the entire region greater than $\delta$ is evaluated from the integration of equation (8) over the different halo mass weighting the comoving number density of dark halos. Indeed, this volume fraction is equivalent to the cumulative distribution function $P(>\delta ; R)$. We thus obtain:

$P(>\delta ; R)=\int_{0}^{\infty} d M V(>\delta ; R, M) n(M)$.

Once provided the cumulative distribution, it is easy to calculate the one-point $\mathrm{PDF}$ from the definition $P(>\delta ; R)=$ $\int_{\delta}^{+\infty} d \delta P(\delta ; R)$ :

$P(\delta ; R)=\left|\frac{d}{d \delta} P(>\delta ; R)\right|$

Equation (10) with equation (9) is the heart of our subsequent analysis. It provides a simple analytic estimate for the degree of non-Gaussian tails, which is, in principle applicable to both weakly non-linear and strongly non-linear regimes. Note that the expression (9) heavily relies on the assumption that any of the dark matter particles reside in each dark halo. This would be true for high density parts of the mass density PDF, but might not be the case for the low density regions, $-1<\delta \lesssim 1$. We shall therefore focus on the high density tails, $\delta \gg 1$. As shown in the following sections, this approach is very useful to understand the essential physical mechanisms that determine the behavior of the high density tail of the PDF.

While the above model prescription is general and does not restrict the choice of the smoothing filter, hereafter, we specifically treat the density field smoothed over the top-hat filter function:

$W_{\mathrm{th}}\left(\boldsymbol{r} ; R_{\mathrm{th}}\right)=\frac{3}{4 \pi R_{\mathrm{th}}^{3}} \Theta\left(R_{\mathrm{th}}-|\boldsymbol{r}|\right)$

where the quantity $\Theta(x)$ denotes Heaviside step function.

\section{DEPENDENCES OF POWER SPECTRUM INDEX AND HALO PROFILE}

Before addressing the comparison with N-body simulations, it is instructive to consider how the halo density profile and initial power spectrum alter the non-Gaussian tails of PDF in a simple analytical manner. In this section, simply assuming the power-law model of halo density profile (4) and scale-free initial spectra $P(k) \propto k^{n}$, we here derive an approximate expression for the one-point $\operatorname{PDF} P\left(\delta ; R_{\mathrm{th}}\right)$.

To compute the PDFs, one must first evaluate the volume factor (8), which is given in terms of the characteristic radius 
$r\left(\delta ; R_{\mathrm{th}}, M\right)$. In Appendix A, the exact expressions for top-hat smoothed density profile is presented in the case of power-law profiles (eqs. 26] 27]). The resultant smoothed profiles become constant at an inner region $r \ll R_{\text {th }}$ and show the power-law behavior at $r \gg R_{\text {th }}$ (see left panel of Fig.6). Based on these exact results, in Appendix A, we further obtain the approximate expression for the smoothed density profiles, which enable us to calculate the quantity $r\left(\delta ; R_{\text {th }}, M\right)$ analytically (see eq. 28]):

$\frac{\rho_{\mathrm{smooth}}\left(r ; R_{\mathrm{th}}, M\right)}{\bar{\rho}} \simeq\left\{\begin{array}{ll}\delta_{0}\left(R_{\mathrm{th}} ; M\right) & ; \quad r<\left(\frac{3-\alpha}{3}\right)^{1 / \alpha} R_{\mathrm{th}} \\ \left(\frac{3-\alpha}{3}\right)\left(\frac{r}{R_{\mathrm{th}}}\right)^{-\alpha} \delta_{0}\left(R_{\mathrm{th}} ; M\right) & ; \quad r \geqslant\left(\frac{3-\alpha}{3}\right)^{1 / \alpha} R_{\mathrm{th}}\end{array}\right.$,

where the function $\delta_{0}\left(R_{\mathrm{th}}, M\right)$ represents the maximum value of the smoothed density profile, $\rho_{\mathrm{smoth}}\left(0 ; R_{\mathrm{th}}, M\right) / \bar{\rho}$, and is given by a function of halo mass:

$\delta_{0}\left(R_{\mathrm{th}}, M\right) \equiv \Delta_{\mathrm{vir}}\left(\frac{R_{\mathrm{th}}}{r_{\mathrm{vir}}}\right)^{-\alpha}=\Delta_{\mathrm{vir}}\left(\frac{M_{R}}{M}\right)^{-\alpha / 3}$,

The quantity $M_{R}$ means the effective halo mass inside the smoothing radius $R_{\mathrm{th}}, M_{R} \equiv(4 \pi / 3) \bar{\rho} \Delta_{\mathrm{vir}} R_{\mathrm{th}}^{3}$. For a given smoothing length $R_{\mathrm{th}}$, equation (13) relates the mass of a halo with its characteristic smoothed density. Adopting the above approximation (12), one can easily solve (7), which yields

$r\left(\delta ; R_{\mathrm{th}}, M\right)=\left\{\begin{array}{ll}0 & ; \delta>\delta_{0}\left(R_{\mathrm{th}} ; M\right) \\ \left\{\frac{3-\alpha}{3} \frac{\delta_{0}\left(R_{\mathrm{th}}, M\right)}{\delta}\right\}^{1 / \alpha} R_{\mathrm{th}} & ; \quad \delta \leqslant \delta_{0}\left(R_{\mathrm{th}} ; M\right)\end{array}\right.$.

Then, with a help of (13), substitution of equation (14) into (8) leads to

$V\left(>\delta ; R_{\mathrm{th}}, M\right)=\left\{\begin{array}{ll}0 & ; \delta>\delta_{0}\left(R_{\mathrm{th}} ; M\right) \\ \left(\frac{3-\alpha}{3} \frac{\Delta_{\mathrm{vir}}}{\delta}\right)^{3 / \alpha} \frac{M}{\bar{\rho} \Delta_{\mathrm{vir}}} \quad ; \quad \delta \leqslant \delta_{0}\left(R_{\mathrm{th}} ; M\right)\end{array}\right.$.

Clearly, the volume factor decreases as $\delta^{-3 / \alpha}$ and is cut off at the maximum density $\delta_{0}\left(R_{\mathrm{th}} ; M\right)$. This indicates that for a certain value of $\delta$, the dominant contribution to the integral in cumulative distribution $P\left(>\delta ; R_{\mathrm{th}}\right)$ is mainly attributed to the halos whose maximum density is larger than that.

Now, turn to focus on the evaluation of the integral in (9). For the cosmological models with initial scale-free spectra, the rms fluctuation $\sigma_{\mathrm{M}}$ in (更) can be expressed as

$\sigma_{\mathrm{M}}=\delta_{\text {crit }}\left(\frac{M}{M_{*}}\right)^{-(n+3) / 6}$.

Substituting the mass function (1) and the volume factor (15) into (9), the cumulative distribution function is analytically evaluated as follows:

$$
\begin{aligned}
P\left(>\delta ; R_{\mathrm{th}}\right) & =\left(\frac{3-\alpha}{3} \frac{\Delta_{\mathrm{vir}}}{\delta}\right)^{3 / \alpha} \frac{1}{\bar{\rho} \Delta_{\mathrm{vir}}} \int_{\left(\delta / \Delta_{\mathrm{vir}}\right)^{3 / \alpha} M_{R}}^{+\infty} n(M) M d M \\
& =\left(\frac{3-\alpha}{3} \frac{\Delta_{\mathrm{vir}}}{\delta}\right)^{3 / \alpha} G(\delta ; \alpha, n),
\end{aligned}
$$

where

$$
\begin{aligned}
G(\delta ; \alpha, n) & \equiv \frac{1}{\bar{\rho} \Delta_{\mathrm{vir}}} \int_{\left(\delta / \Delta_{\mathrm{vir}}\right)^{3 / \alpha} M_{R}}^{+\infty} n(M) M d M \\
& =\frac{A}{\Delta_{\mathrm{vir}} \sqrt{\pi}} \int_{y_{\mathrm{R}}(\delta) / 2}^{+\infty}\left\{1+\frac{1}{(2 x)^{p}}\right\} x^{1 / 2} e^{-x} \frac{d x}{x} \\
& =\frac{A}{\Delta_{\mathrm{vir}} \sqrt{\pi}}\left\{\Gamma\left(\frac{1}{2}, \frac{y_{\mathrm{R}}(\delta)}{2}\right)+2^{-p} \Gamma\left(\frac{1}{2}-p, \frac{y_{\mathrm{R}}(\delta)}{2}\right)\right\}
\end{aligned}
$$

with the function $\Gamma(a, x)$ being the incomplete Gamma function, $\Gamma(a, x) \equiv \int_{x}^{\infty} d t t^{a-1} e^{-t}$. Here, the function $y_{\mathrm{R}}(\delta)$ is defined by

$y_{\mathrm{R}}(\delta) \equiv a\left(\frac{M_{R}}{M_{*}}\right)^{(n+3) / 3}\left(\frac{\delta}{\Delta_{\text {vir }}}\right)^{(n+3) / \alpha}=a\left(\frac{\delta}{\delta_{*}}\right)^{(n+3) / \alpha}$,

where $\delta_{*} \equiv \delta_{0}\left(R_{\mathrm{th}}, M_{*}\right)$ and denotes the characteristic smoothed density of the halo with mass $M_{*}$, which play a key role to reveal the sensitivity of the non-Gaussian tails.

Equation (17) indicates that a shape of the PDF is determined by the combination of two contributions: One is the halo profile. The scaling $\delta^{-3 / \alpha}$ in equation (17) reflects the slope of the halo profile. The flatter the halo profile is, the more rapidly the volume factor varies, thus the steeper the PDF becomes. Another contribution is the shape of the mass function, since 

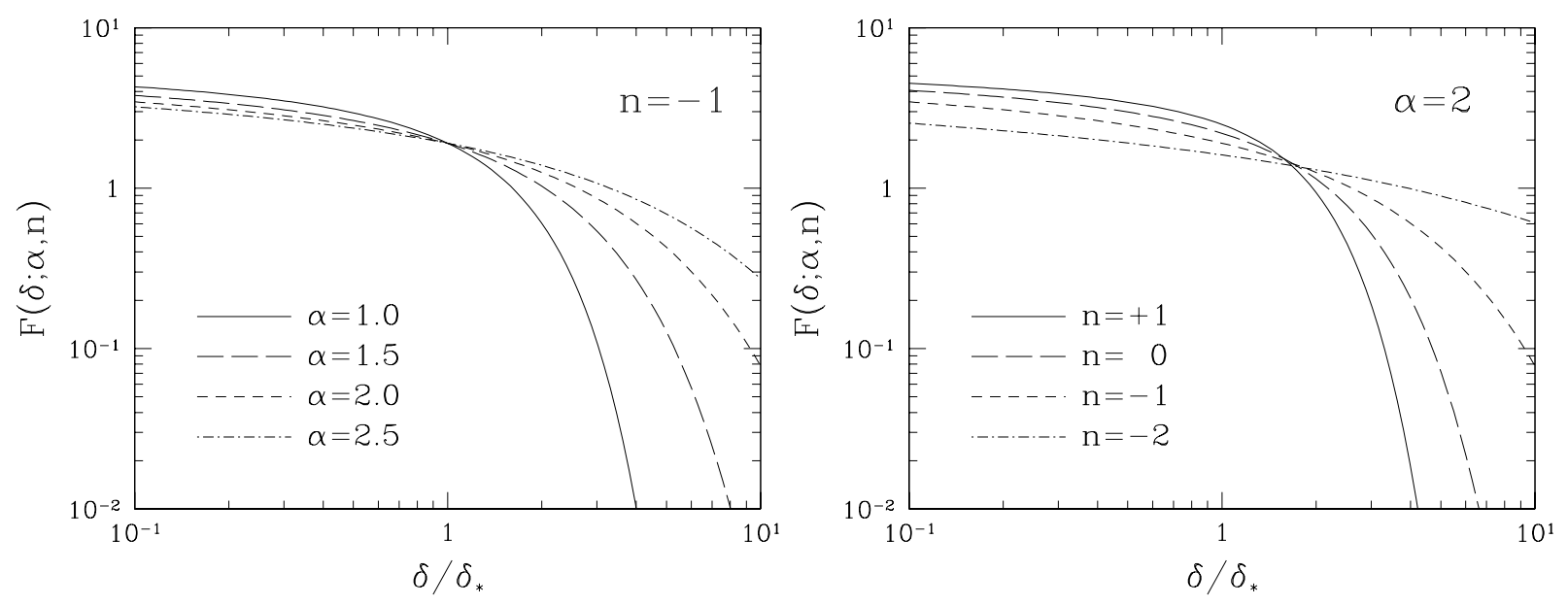

Figure 1. $F(\delta, \alpha, n)$ defined by eq.(21) as a function of $\delta / \delta_{*}$. Dependence of the halo density profiles (left) and initial spectra(right).

the factor $M / \bar{\rho} \Delta_{\text {vir }}$ gives the characteristic volume of the halo with the mass $M$, and the lower limit of the integration in equation (18) is, roughly speaking, determined by the halo mass whose characteristic smoothed density (defined by eq. 13 ) just coincides with $\delta$. Therefore, the function $G(\delta ; \alpha, n)$ gives the volume fraction of halos (i.e., a sum of characteristic volume of halos) whose mass is larger than such lower limit, and directly reflects the shape of the halo mass function. Note that the dependence of the spectral index $n$ on the PDF enters only through the function $G(\delta ; \alpha, n)$.

Now from 117 , the one-point $\operatorname{PDF} P\left(\delta ; R_{\mathrm{th}}\right)$ is analytically expressed as follows:

$P\left(\delta ; R_{\mathrm{th}}\right)=\frac{3}{\alpha}\left(\frac{3-\alpha}{3}\right)^{3 / \alpha} \frac{1}{\Delta_{\mathrm{vir}}}\left(\frac{\delta}{\Delta_{\mathrm{vir}}}\right)^{-(3 / \alpha+1)} F(\delta ; \alpha, n)$,

with

$$
\begin{aligned}
F(\delta ; \alpha, n) & =G(\delta ; \alpha, n)+\frac{\alpha}{3} \Delta_{\mathrm{vir}}\left|\frac{d}{d \delta} G(\delta ; \alpha, n)\right| \\
& =\frac{A}{\Delta_{\mathrm{vir}} \sqrt{\pi}}\left[\Gamma\left(\frac{1}{2}, \frac{y_{\mathrm{R}}(\delta)}{2}\right)+2^{-p} \Gamma\left(\frac{1}{2}-p, \frac{y_{\mathrm{R}}(\delta)}{2}\right)+\frac{n+3}{3 \sqrt{2}}\left\{y_{\mathrm{R}}(\delta)\right\}^{1 / 2}\left(1+\frac{1}{\left\{y_{\mathrm{R}}(\delta)\right\}^{p}}\right) e^{-y_{\mathrm{R}}(\delta) / 2}\right]
\end{aligned}
$$

The expression (20) with (19) and (21) is a main analytical result in this paper. Qualitatively, it reveals the strong dependence of the halo density profiles and the sensitivity to the initial spectra.

Figure 1 shows the behavior of the function $F(\delta ; \alpha, n)$ for various values of $n$ (left panel) and for $\alpha$ (right panel). Note again that the dependence of the power index $n$ on the PDF enters only through the function $F(\delta ; \alpha, n)$. It is clear from both panels that the slope of the function $F$ becomes sensitive to both $n$ and $\alpha$ in the range $\delta / \delta_{*}>1$, corresponding to the mass range $M \gtrsim M_{*}$. The sensitivity to the power index $n$ simply results from the steep exponential tails of the halo mass function, which becomes very sensitive to the spectral index in such mass range. And the sensitivity to the slope of the halo profile $\alpha$ is also explained from the exponential tails of mass function, that is, the variation in the relation between characteristic smoothed density $\delta_{0}$ (eq. 13 ) and halo mass $M$ due to a small change of $\alpha$ is significantly enhanced through such exponential dependences. On the other hand, the slope of the function $F$, in turn, becomes insensitive to both $n$ and $\alpha$ at the region $\delta / \delta_{*}<1$. This is because in such range (corresponding to the mass range of $M<M_{*}$ ) the mass function only weakly depends on the spectral index $n$ and scales as $n(M) \propto M^{(n-9) / 6}$. Therefore, within the range $\delta / \delta_{*}<1$, the PDF is almost solely determined by the contribution from the halo profile and varies as $\propto \delta^{-(3 / \alpha+1)}$, while at the region $\delta / \delta_{*}>1$, the function $F$ varies much faster than $\delta^{-(3 / \alpha+1)}$, thus the shape of PDF is determined by the contribution from the exponential tails of mass function.

Figure 2 shows the approximate expression of one-point PDF (20) for various slope of the halo profiles (left) and for various initial spectra (right). For definiteness, we assume the Einstein-de Sitter universe (density parameter $\Omega_{0}=1$, cosmological constant $\lambda_{0}=0$ ) and the non-linear mass $M_{*}$ in each model is determined by introducing the artificial length scale $L_{\mathrm{box}}$ and normalizing the rms fluctuation $\sigma_{\mathrm{M}}$ to unity at $R_{M}=0.1 L_{\mathrm{box}}$. The smoothing radii are specifically chosen as both the large $\delta_{*}(\sim 30-90)\left(R_{\mathrm{th}}=0.02 L_{\mathrm{box}}\right)$ and the small $\delta(\sim 0.3-1.5)\left(R_{\mathrm{th}}=0.15 L_{\mathrm{box}}\right)$. Table 1 summarizes the values of $\delta_{*}$ for the models shown in Figure 2 .

In left panel, the spectral index are fixed to $n=-1$ but the slope of halo profile $\alpha$ varies from 1 to 2.5 , while in right panel, $\alpha$ is fixed to 2 but $n$ varies from -2 to +1 . It is clear from the cases of $R_{\text {th }}=0.02 L_{\text {box }}$ in both panels that at the region $\delta \lesssim \delta_{*}$, the slope of PDF hardly depends on $n$ but sensitively depends on $\alpha$. On the other hand, at $\delta \gtrsim \delta_{*}$ (see the cases of $\widetilde{R}_{\mathrm{th}}=0.02 L_{\mathrm{box}}$ in the right panel), the shape of PDF strongly depends on $n$. It follows from these results that if one takes smaller smoothing scale than the virial radius of the halo mass $M_{*}$ (thus $\delta_{*} \gtrsim 100$ ), the slope of the PDF almost solely determined by the slope of the halo profile at the intermediate range of $5<\delta<50$, while the shape of PDF strongly depends both on $\alpha$ and $n$ if we conversely take a larger smoothing scale (so that $\delta_{*} \lesssim 10$ ). Figure 3 shows this point clearly. In the 

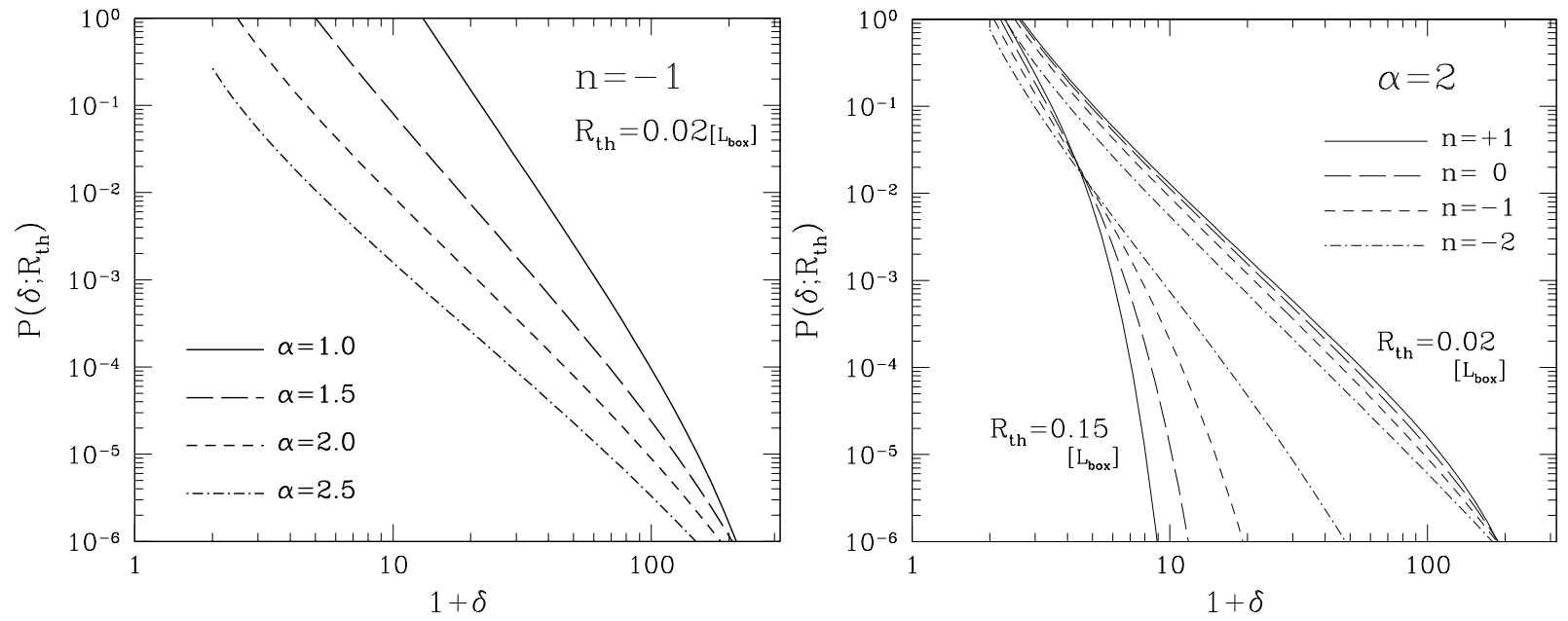

Figure 2. Dependence of the halo density profiles(left) and initial spectra(right) on the approximate expression of the one-point PDF 200 in scale-free models. Here, the normalization of rms mass fluctuation $\sigma_{M}$ is determined by introducing the auxiliary length scale $L_{\text {box }}$ and setting $\sigma_{M}=1$ at $R_{M}=0.1 L_{\text {box }}$ in each panel. While the initial spectrum and the top-hat smoothing radius are respectively chosen as $n=-1$ and $R_{\mathrm{th}}=0.02 L_{\mathrm{box}}$ in left panel, the right panel fixes the slope of the power-law profile to $\alpha=2.0$.

Table 1. The characteristic smoothed density $\delta_{*}$ for models presented in Figure 目.

\begin{tabular}{cccccc}
\hline \multicolumn{2}{c}{ Left panel } & \multicolumn{4}{c}{ Right Panel } \\
\multicolumn{2}{c}{$n=-1, R_{\mathrm{th}}=0.02\left[L_{\mathrm{box}}\right]$} & \multicolumn{2}{c}{$\alpha=2, R_{\mathrm{th}}=0.02\left[L_{\mathrm{box}}\right]$} & \multicolumn{2}{c}{$\alpha=2, R_{\mathrm{th}}=0.15\left[L_{\mathrm{box}}\right]$} \\
$\alpha$ & $\delta_{*}$ & $n$ & $\delta_{*}$ & $n$ & $\delta_{*}$ \\
\hline \hline 1.0 & 93.7 & +1 & 83.3 & +1 & 1.48 \\
1.5 & 68.0 & 0 & 70.0 & 0 & 1.24 \\
2.0 & 49.4 & -1 & 49.4 & -1 & 0.88 \\
2.5 & 35.9 & -2 & 17.4 & -2 & 0.31 \\
\hline
\end{tabular}

figure, the PDF in the case of $n=-1$ and $\alpha=2$ is shown restricting the contribution of halo mass to some small mass ranges. It turns out that at $\delta>\delta_{*}$, the major contribution to the PDF at a certain value of $\delta$ comes from halos whose characteristic mass scale $\delta_{0}$ roughly coincides with $\delta$, thus the shape of PDF reflects shape of the halo mass function. At $\delta<\delta_{*}$, however, halos with $\delta_{0}=\delta$ no longer lead to a major contribution, but halos whose characteristic density $\delta_{0}$ is larger than $\delta$ instead contribute to the PDF. In this case, PDF reflects the profile of the halos.

\section{COMPARISON WITH N-BODY SIMULATIONS}

We are now in a position to compare the one-point PDFs from the dark halo approach with those obtained from N-body simulations. To investigate this issue, the analytic results in the previous section turn out to become inadequate because of the non power-law nature of the realistic halo profiles. Hence, all the model predictions presented below are based on the NFW profile (2). In computing the analytic PDF (10), the top-hat smoothed halo $\rho_{\text {smooth }}$ is numerically evaluated in the Fourier space, in which we use the following fitting function $y(k ; M)$ for the Fourier transform of the NFW profile $\rho_{\mathrm{NFw}} / \bar{\rho}(\mathrm{Ma} \&$ Fry 2000):

$y(k ; M)=\frac{4 \pi}{3} \delta_{c} r_{s}^{3} \frac{\ln (e+1 / q)-\ln [\ln (e+1 / q)]}{\left(1+q^{1.1}\right)^{(2 / 1.1)}}$,

with $q=k r_{s}$.

For the present purpose, we specifically treat the N-body simulation data for the scale-free models with initial power spectra $P(k) \propto k^{n}(n=1,0,-1,-2)$ (Jing 1998) and the CDM model with cosmological constant(Lambda CDM, hereafter LCDM; Jing \& Suto 1998). All the models employ $N=256^{3}$ dark matter particles in a periodic comoving cube, $L_{\mathrm{b} o x}^{3}$, where the box size of the LCDM model is specifically chosen as $L_{\mathrm{box}}=100 h^{-1} \mathrm{Mpc}$. The gravitational force calculation is based on the $\mathrm{P}^{3} \mathrm{M}$ algorithm. While the scale-free models assume an Einstein-de Sitter universe, cosmological parameters of the LCDM model are chosen as $\left(\Omega_{0}, \lambda_{0}, h, \sigma_{8}\right)=(0.3,0.7,0.7,1.0)$, where the normalization $\sigma_{8}$ means the linear rms fluctuation 


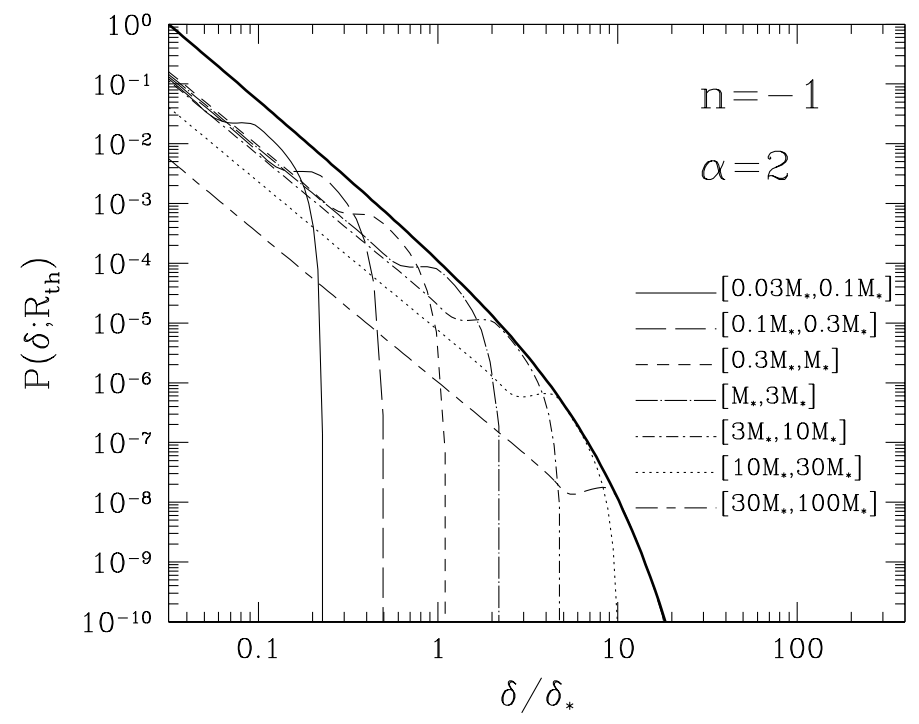

Figure 3. Contribution to the tails of PDF from different halo mass range for the model with $n=-1$ and $\alpha=2$. The thick solid lines are the exact calculation of equation (10) with (9), while the thin lines represent the results restricting the range of integration. Here, the smoothing radius is specifically chosen as $R_{\mathrm{th}}=0.02\left[L_{\mathrm{box}}\right]$, but the the horizontal scaling $\delta / \delta_{*}$ is not affected by any specific choice of $R_{\mathrm{th}}$.

Table 2. The estimated values of the rms fluctuation amplitude $\sigma\left(R_{\mathrm{th}}\right)$ in Figure日 from the simulation data (labeled $\sigma_{\text {sim }}$, the non-linear fitting formula by Peacock \& Dodds (1996) (labeled by $\sigma_{\mathrm{PD}}$ ) and $\delta_{*}$.

\begin{tabular}{cccccccc}
\hline & \multicolumn{3}{c}{ LCDM } & & \multicolumn{3}{c}{ scale-free $(n=-1)$} \\
$R_{\text {th }}\left[h^{-1} \mathrm{Mpc}\right]$ & $\sigma_{\text {sim }}$ & $\sigma_{\text {PD }}$ & $\delta_{*}$ & $R_{\text {th }}\left[L_{\text {box }}\right]$ & $\sigma_{\text {sim }}$ & $\sigma_{\text {PD }}$ & $\delta_{*}$ \\
\hline \hline 2 & 4.17 & 4.08 & 11.0 & 0.02 & 4.81 & 4.99 & 35.8 \\
6 & 1.37 & 1.40 & 0.569 & 0.05 & 1.80 & 1.95 & 3.25 \\
18 & 0.44 & 0.50 & 0.0272 & 0.15 & 0.54 & 0.64 & 0.165 \\
\hline
\end{tabular}

at $R_{\mathrm{th}}=8 h^{-1} \mathrm{Mpc}$. As for the scale-free models, the normalization of the density fluctuation is determined by setting the linear rms fluctuation to unity at $R_{\mathrm{th}}=0.1 L_{\mathrm{box}}$.

Figure 1 shows the N-body results of the one-point PDF for the top-hat smoothed density field in various smoothing radii (crosses). The left panel shows the results in LCDM model, while the right panel plots the PDFs in the scale-free model with the initial spectrum $n=-1$. The error-bars in the left panel indicate the $1-\sigma$ errors among three different realizations. The smoothing radii in left(right) panel are chosen as $R_{\mathrm{th}}=2,6,18 h^{-1} \mathrm{Mpc}\left(R_{\mathrm{th}}=0.02,0.05,0.15 L_{\mathrm{box}}\right)$, which are typically scaled from non-linear to weakly non-linear regime. In Table 2, computed values of the rms amplitude $\sigma$ are presented(labeled by $\left.\sigma_{\text {sim }}\right)$. Obviously, the degree of non-linearity becomes significant as decreasing $R_{\text {th }}$ and the resultant PDFs show much longer non-Gaussian tails.

In Figure 4, the one-point PDFs from the dark halo approach are shown in solid lines. Within the validity range $\delta \gtrsim 1$, in both panels, the dark halo approach reproduces the non-Gaussian tails of simulated PDF reasonably well. The predictions at large smoothing radius $R_{\mathrm{th}}=18 h^{-1} \mathrm{Mpc}\left(0.15 L_{\mathrm{box}}\right)$ somewhat over-predict the simulations, however, as have been pointed out by Kayo et al.(2001), this is most likely due to the finite volume effect of the N-body simulations.

For comparison, the dashed lines in Figure 1 show the empirical log-normal distribution. The one-point PDF of the log-normal model is analytically expressed as

$P_{\mathrm{LN}}(\delta)=\frac{1}{\sqrt{2 \pi \sigma_{\mathrm{LN}}^{2}}} \exp \left\{-\frac{\left[\log (1+\delta)+\sigma_{\mathrm{LN}}^{2} / 2\right]^{2}}{2 \sigma_{\mathrm{LN}}^{2}}\right\} \frac{1}{1+\delta}$,

where the single parameter $\sigma_{\mathrm{LN}}$ is related to the rms amplitude of density fluctuation at a given smoothing radius $R_{\mathrm{th}}, \sigma\left(R_{\mathrm{th}}\right)$ :

$\sigma_{\mathrm{LN}}^{2}=\log \left[1+\sigma^{2}\left(R_{\mathrm{th}}\right)\right]$

For a given set of cosmological parameters, the quantity $\sigma\left(R_{\mathrm{th}}\right)$ is computed using a fitting formula of non-linear power spectrum by Peacock \& Dodds (1996) or even by the dark halo approach (Seljak 2000; Ma \& Fry 2000; Scoccimarro et al. 2001; Hamana, Yoshida \& Suto 2001). The resultant values are summarized in Table 2 (labeled by $\sigma_{\mathrm{PD}}$ ) and adopting those, 

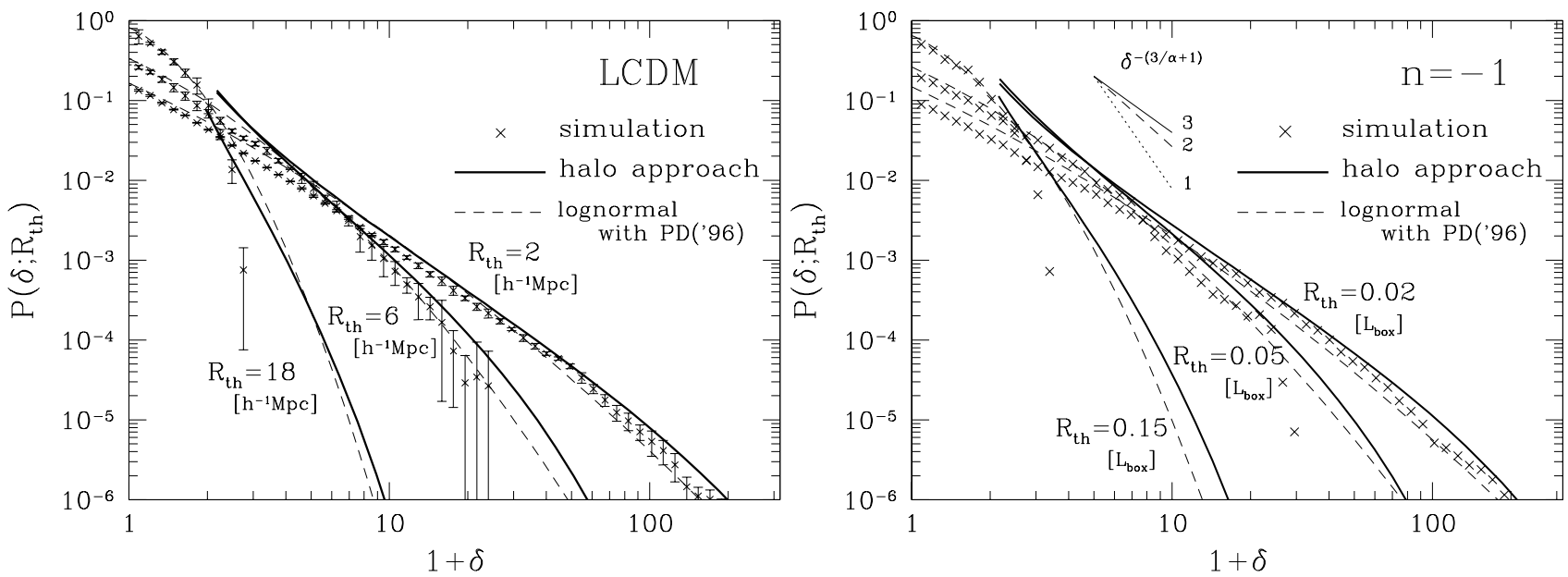

Figure 4. Comparison of one-point PDF between analytic models (solid, dashed) and N-body simulations(crosses) in LCDM model(left) and scale-free model with $n=-1$ (right). In each panel, the results from dark halo approach are shown in solid lines, while the dashed lines indicate the log-normal model prediction (23). In plotting the log-normal PDF, the rms fluctuation of density field $\sigma$ is evaluated using the fitting formula by Peacock \& Dodds (1996). The error-bars in left panel denote the 1- $\sigma$ errors among three different realization of simulation data. Also, in the right panel, the asymptotic slopes of the dependence $\delta^{-(\alpha / 3+1)}$ with $\alpha=1,2$, and 3 are shown.
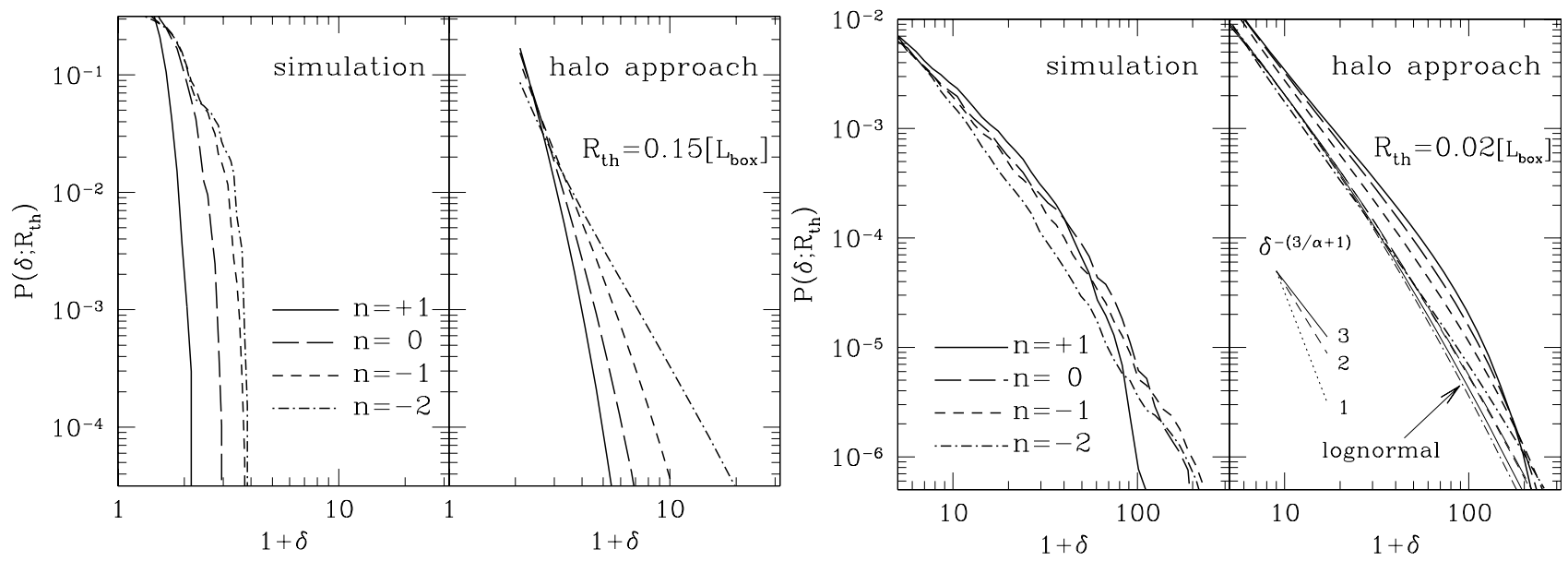

Figure 5. Comparison of non-Gaussian tails of PDF between dark halo approach and N-body simulations in cases with scale-free initial spectra. The left(right) panel depicts the results at the smoothing radius $R_{\mathrm{th}}=0.02 L_{\mathrm{box}}\left(R_{\mathrm{th}}=0.15 L_{\mathrm{box}}\right)$. For comparison, we also plot the log-normal PDFs in the right-side of the right panel (thin lines), where rms fluctuation of log-normal PDF, $\sigma$ is calculated from simulations. Also, the asymptotic slope of the dependence $\delta^{-(\alpha / 3+1)}$ with $\alpha=1,2$, and 3 are shown.

the log-normal predictions (23) are plotted. In this sense, both empirical and analytic models in Figure 1 provide a fairly self-consistent prediction without invoking any information of simulation data.

As mentioned in Kayo et al. (2001), the rms amplitude $\sigma_{\mathrm{PD}}$ takes a slightly larger value than that of the simulation, $\sigma_{\mathrm{sim}}$, which should be ascribed to the finite box-size of the simulations. Apart from this small discrepancy, the log-normal PDFs approximate the simulated PDF fairly accurately in both low-density and high-density regions. The most striking evidence is that the tails of analytic PDF almost resembles those of the log-normal PDF, which is also consistent with the prediction from perturbation theory(Bernardeau 1994b; Bernardeau \& Kofman 1995). According to the Table 月, the theoretical estimate of characteristic smoothed density $\delta_{*}$ roughly varies from $\delta_{*} \sim 0.03$ to 40 as decreasing the smoothing radius. Thus, the tails of PDF at smoothing radius $R_{\mathrm{th}}=2 h^{-1} \mathrm{Mpc}$ or $0.02 L_{\mathrm{box}}$ basically reflect the shape of the halo profiles and they scale as $\delta^{-(\alpha / 3+1)}$. Comparing the shape of PDFs with the slope of the dependence $\delta^{-(\alpha / 3+1)}$ superimposed in the right panel of Figure $\mathrm{A}$, the non-Gaussian tails at smaller smoothing radii can be regarded as a consequence of outer slope of the NFW profile, $\alpha=3$.

Figure : shows weak and strong sensitivity of the non-Gaussian tails to the initial spectrum in scale-free models. Specifically choosing the smoothing radius as $R_{\mathrm{th}}=0.15 L_{\mathrm{box}}(l e f t)$ and $R_{\mathrm{th}}=0.02 L_{\mathrm{box}}($ right $)$, the results from the dark halo approach are plotted in right-side of each panel, while the left-side shows the simulation results. For comparison, the log-normal PDFs are also plotted in right-side of the right panel(thin-lines), where rms amplitude $\sigma$ is calculated from simulations. In cases with large smoothing radius $R_{\mathrm{th}}=0.15 L_{\mathrm{box}}$, the simulated PDFs rapidly fall off and they eventually vanish, while the analytic 
model of PDF shows a slightly longer non-Gaussian tail. This discrepancy is basically ascribed to the finite volume size of the simulations (Kayo et al. 2001), however, the tendency seen in both panels is qualitatively similar. The analytic model reproduces the systematic behavior for the variation of spectral index $n$ (see also the right-panel of Fig.2). On the other hand, for smaller smoothing radius with $R_{\mathrm{th}}=0.02 L_{\mathrm{box}}$, except for the noisy data of scale-free model with $n=+1$, both left- and right-side of the panel show not only qualitatively but also quantitatively similar dependence on the initial power spectra. Again, the asymptotic behavior of the PDF is quite similar to the results with steeper power-law profile $\alpha \sim 3$ (compare with the slope $\delta^{-(\alpha / 3+1)}$ superimposed in the figure). As a consequence, the non-Gaussian tails of PDF seem to resemble the log-normal PDF insensitive to the initial spectrum, as has been reported by Kayo et al. (2001). At a closer look at tails of PDF, however, the simulated PDFs still exhibit the initial spectrum dependence and they slightly deviate from log-normal PDFs (thin-lines). In contrast, the analytic PDF from the dark halo approach successfully reproduces this weak dependence. Note that the theoretical estimate of the characteristic smoothed density yields $\delta_{*} \sim 10-70$ at $R_{\mathrm{th}}=0.02 L_{\mathrm{box}}$, which suggests that the the simulated PDF will show a strong sensitivity to the spectral index at the high density tail $\delta / \delta_{*} \gg 1$, and thereby a large discrepancy between log-normal prediction and simulation is expected. Interestingly, there is some indication at the region $\delta \gtrsim 200$ in both simulation and halo approach, where the systematic variation for $n$ apparently ceases.

Therefore, we conclude that the analytic model based on the dark halo approach reasonably predicts the general tendency in both weakly and strongly non-linear regime and it quantitatively provides a useful approximation to the non-Gaussian tails of simulated PDF in the strongly non-linear regime.

\section{DISCUSSION \& CONCLUSION}

In this paper, utilizing the dark halo approach, we have presented a simple analytic model characterizing the non-Gaussian tails of one-point PDF and investigated the sensitivity to both the initial power spectrum and the halo density profiles in the non-linear regime. Assuming the power-law profile of dark halos and the scale-free initial spectrum, the approximate expression for the tails of PDF is analytically derived (eq.20 with 19 21]). Using this result, we explained the sensitivity to the initial spectrum seen in the simulation and the significant dependences on the halo profiles. Further, quantitative comparison with N-body simulation was made, which leads to the conclusion that the analytic model reasonably reproduces the simulated PDF qualitatively and quantitatively in a strongly non-linear regime. Then, the tails of simulated PDF turn out to resemble the log-normal distribution (23) at the region $\delta \lesssim 100$ in a strongly non-linear regime.

In our model, the key quantity is the characteristic smoothed density $\delta_{*}=\rho_{\text {smooth }}\left(0 ; R, M_{*}\right) / \bar{\rho}$, which discriminates the weak/strong sensitivity to the halo profile and initial power spectrum (see eq. 19]). At $\delta / \delta_{*} \lesssim 1$, the tails of PDF basically follow the halo profile and varies as $\delta^{-(3 / \alpha+1)}$, while the PDF at $\delta / \delta_{*} \gtrsim 1$ exhibits a strong sensitivity to the initial spectrum, which can be ascribed to the steep exponential tails of halo mass function. In CDM models at present, the characteristic smoothed density typically takes the value $\delta_{*} \gg 1$ for a small smoothing scale $R_{\mathrm{th}} \sim 1 \mathrm{Mpc} / h$ and conversely $\delta_{*} \ll 1$ for a large smoothing scale $R_{\mathrm{th}}>10 \mathrm{Mpc} / h$. Therefore, the log-normal behavior seen in the non-linear regime can be regarded as a consequence of the universality of the halo density profile. Further considering the fact that the slope of the simulated PDF is similar to that of the analytic results with $\alpha \simeq 3$, the weak sensitivity to the initial spectrum is especially due to the universal outer profile of $\rho \propto r^{-3}$.

The qualitative feature in the dark matter PDFs might be also the case for the PDF of galaxies, which has been known to be well approximated by the log-normal PDF (e.g., Hamilton 1985; Bouchet et al. 1993; Kofman et al. 1994) as the density PDF is. The number density of galaxies within the clusters is known to be fitted well by the King model (King 1962), which has a radial profile of $\rho_{g} \propto\left[1+\left(r / r_{c}\right)^{2}\right]^{-3 / 2}$, where $r_{c}$ is a core radius of order $0.1 h^{-1} \mathrm{Mpc}$ (Wu \& Hammer 1993 and references therein). Thus, on suitably larger smoothing scale $R_{\text {th }} \gtrsim r_{c}$, smoothed density profile becomes $\rho_{g} \propto r^{-3}$, at an outer part. Therefore, it is very likely that the similarity in the shape of the PDF tails between the dark matter and the galaxy is due to simply their similar distribution within halos.

Further, the good agreement in the non-Gaussian tails of density PDF between the halo approach prediction and N-body data indicates that the halo model is not only used to qualitative study but also allows us to make a quantitative prediction. Although the dark matter PDF is hard to measure, it may be applied to related measurements such like the weak lensing convergence which is a line-of-sight projection of the matter density weighted by the lensing efficiency and is obtained from a coherent distortion in distant galaxy images (see Mellier 1999; Bartelmann \& Schneider 2000 for reviews). Taruya et al. (2002) showed that the lensing convergence PDF is well approximated by the log-normal model and pointed out that the lensing convergence field can be regarded as a simple projection of the density field. This implies that one can safely apply the halo approach to the lensing convergence PDF. Actually, Kruse \& Schneider (2000) employed the halo approach to predict the non-Gaussian tail in PDF of lensing convergence arguing it may be used to discriminate the cosmological model. Their prediction was tested against numerical experiments and a good agreement was found (Reblinsky et al. 1999). Since the approach adopted by Kruse \& Schneider (2000) is essentially the same as described in this paper, one naturally expects that the tails of lensing convergence PDF basically follows the outer slope of the halo profiles at smaller angular scale $\theta \sim 1^{\prime}-5^{\prime}$, and the log-normal behavior found by Taruya et al. (2002) can be explained by similar reason to the mass density PDF.

Therefore, as far as the CDM model with a Gaussian initial condition is concerned, theoretical prediction based on the dark halo approach consistently explains the log-normal nature of observable clustering statistics as a common feature in the hierarchical clustering universe. This conversely indicates that the statistics related to the non-Gaussian tails of PDF provide a powerful cosmological probe to the nature of dark matter clustering, which enables us to constrain the number density and 
the density profile of dark halos. Probably, one promising application is the weak lensing statistics, as has been demonstrated by Kruse \& Schneider (2000). Recently, from the $2.1 \mathrm{deg}^{2}$ image taken with a wide field camera on the prime focus of Subaru Telescope, non-Gaussian signature of the lensing convergence was detected evaluating the peak distribution function (Miyazaki et al. 2002). The number count of high positive peaks, which are thought to come from the dark halos, was compared with the theoretical prediction using halo approach and showed a consistent result with NFW profiles under the LCDM cosmology. Note, however, that the analytic models of PDF from the dark halo approach currently limit the applicability to highly nonGaussian tails in a strongly nonlinear regime. To obtain a more stringent constraint on the cosmological parameter and/or the number density of dark halos, we should further improve the applicability to the low-density region up to the observable scale of the non-linear regime. In the light of this, the perturbative construction of PDF by Bernardeau \& Valageas (2000) or the non-perturbative modeling from the hierarchical ansatz (Munshi \& Jain 2000; Valageas 2000), as well as the empirical log-normal model (Taruya et al.2002) could be useful and be regarded as a complementary approach. Combining the present work with these approaches would be possible to improve the prediction dramatically and will be discussed elsewhere.

\section{ACKNOWLEDGMENTS}

We thank Y.P.Jing for kindly providing us his N-body data, Y.Suto for reading of the manuscript and critical comments, M.Takada and K.Yoshikawa for valuable discussion. I.K is supported by Takenaka-Ikueikai Fellowship. T.H acknowledges supports from Japan Society for Promotion of Science (JSPS) Research Fellowships. A.T is supported by the grand-in-aid for Scientific Research of JSPS (No.1470157).

\section{APPENDIX A: SMOOTHED DENSITY PROFILE FOR POWER-LAW HALOS}

In this appendix, adopting the top-hat filter function (11), we derive the exact expression for the smoothed halo profiles. Substituting the definition (11) into (6), the expressions for smoothed density profile can be reduced to

$$
\begin{aligned}
\rho_{\text {smooth }}\left(r ; R_{\mathrm{th}}, M\right) & =\frac{3}{4 \pi R_{\mathrm{th}}^{3}} \int d^{3} \boldsymbol{r}^{\prime} \Theta\left(R_{\mathrm{th}}-\left|\boldsymbol{r}^{\prime}\right|\right) \rho\left(\left|\boldsymbol{r}-\boldsymbol{r}^{\prime}\right| ; M\right) \\
& =\frac{3}{2 R_{\mathrm{th}}^{3}} \int_{0}^{R_{\mathrm{th}}} d s s^{2} \int_{-1}^{1} d x \rho\left(\sqrt{r^{2}+s^{2}-2 r s x} ; M\right) .
\end{aligned}
$$

For the power-law profiles (4), all the integrals in the expression (25) are analytically evaluated, which lead to

$$
\begin{aligned}
\rho_{\mathrm{smooth}}\left(r ; R_{\mathrm{th}}, M\right)= & \frac{3 \delta_{c} \bar{\rho} r_{s}^{\alpha}}{2 R_{\mathrm{th}}^{3}} \int_{0}^{R_{\mathrm{th}}} d s s^{2} \frac{|r-s|^{2-\alpha}-(r+s)^{2-\alpha}}{(\alpha-2) r s} \\
= & \frac{3}{2(\alpha-2)} \rho_{\text {power }}\left(R_{\mathrm{th}} ; M\right) \\
& \quad \times\left\{\begin{array}{l}
\frac{1}{3-\alpha}\left\{(1-u)^{3-\alpha}+(1+u)^{3-\alpha}\right\}+\frac{u^{-1}}{4-\alpha}\left\{(1-u)^{4-\alpha}-(1+u)^{4-\alpha}\right\} \quad ; \quad\left(0<r \leqslant R_{\mathrm{th}}\right) \\
\frac{1}{3-\alpha}\left\{(1+u)^{3-\alpha}-(u-1)^{3-\alpha}\right\}+\frac{u^{-1}}{4-\alpha}\left\{(u-1)^{4-\alpha}-(1+u)^{4-\alpha}\right\} \quad ; \quad\left(R_{\mathrm{th}}<r\right)
\end{array}\right.
\end{aligned}
$$

Here, the variable $u$ denotes $r / R_{\mathrm{th}}$. Notice that the above expressions are valid for the profiles with $\alpha \neq 2$. For the power-law profile with $\alpha=2$ (singular isothermal sphere), the logarithmic dependence of the radius $r$ appears, which can be separately evaluated as follows:

$\rho_{\text {smooth }}\left(r ; R_{\mathrm{th}}, M\right)=\frac{3 \delta_{c} \bar{\rho} r_{s}^{2}}{2 R_{\mathrm{th}}^{3}} \int_{0}^{R_{\mathrm{th}}} d s \frac{s}{r} \log \left|\frac{r+s}{r-s}\right|=\frac{3}{2} \rho\left(R_{\mathrm{th}} ; M\right)\left\{1+\frac{1}{2}\left(u^{-1}-u\right) \log \left|\frac{u+1}{u-1}\right|\right\}$.

While the above results are exact, it is convenient and useful to derive the simple analytic expressions for the one-point PDF $P\left(\delta ; R_{\mathrm{th}}\right)$ (see Sec. 3$)$. From the asymptotic behavior of the smoothed halo profiles, we approximate the expressions 26 and (27) as follows:

$\rho_{\text {smooth }}\left(r ; R_{\mathrm{th}}, M\right) \simeq \begin{cases}\left(\frac{3}{3-\alpha}\right) \rho_{\text {power }}\left(R_{\mathrm{th}} ; M\right) & ; r<\left(\frac{3-\alpha}{3}\right)^{1 / \alpha} R_{\mathrm{th}} \\ \left(\frac{r}{R_{\mathrm{th}}}\right)^{-\alpha} \rho_{\text {power }}\left(R_{\mathrm{th}} ; M\right) & ; \quad r \geqslant\left(\frac{3-\alpha}{3}\right)^{1 / \alpha} R_{\mathrm{th}}\end{cases}$

The left panel of Figure 8 compares the approximation (28) with the exact results 26 and (27). Apart from the intermediate scales $r / R_{\mathrm{th}} \sim 1$, the approximation recovers the exact results reasonably well. On the other hand, deviation at the intermediate scale slightly increases as increasing the slope of profile $\alpha$, which leads to the under-prediction of volume factor $V\left(>\delta ; R_{\mathrm{th}}, M\right)$. Hence, for steeper halo profile $\alpha \sim 3$, the analytic PDF using (28) tends to under-predict the amplitude of PDF as shown in the right panel. 

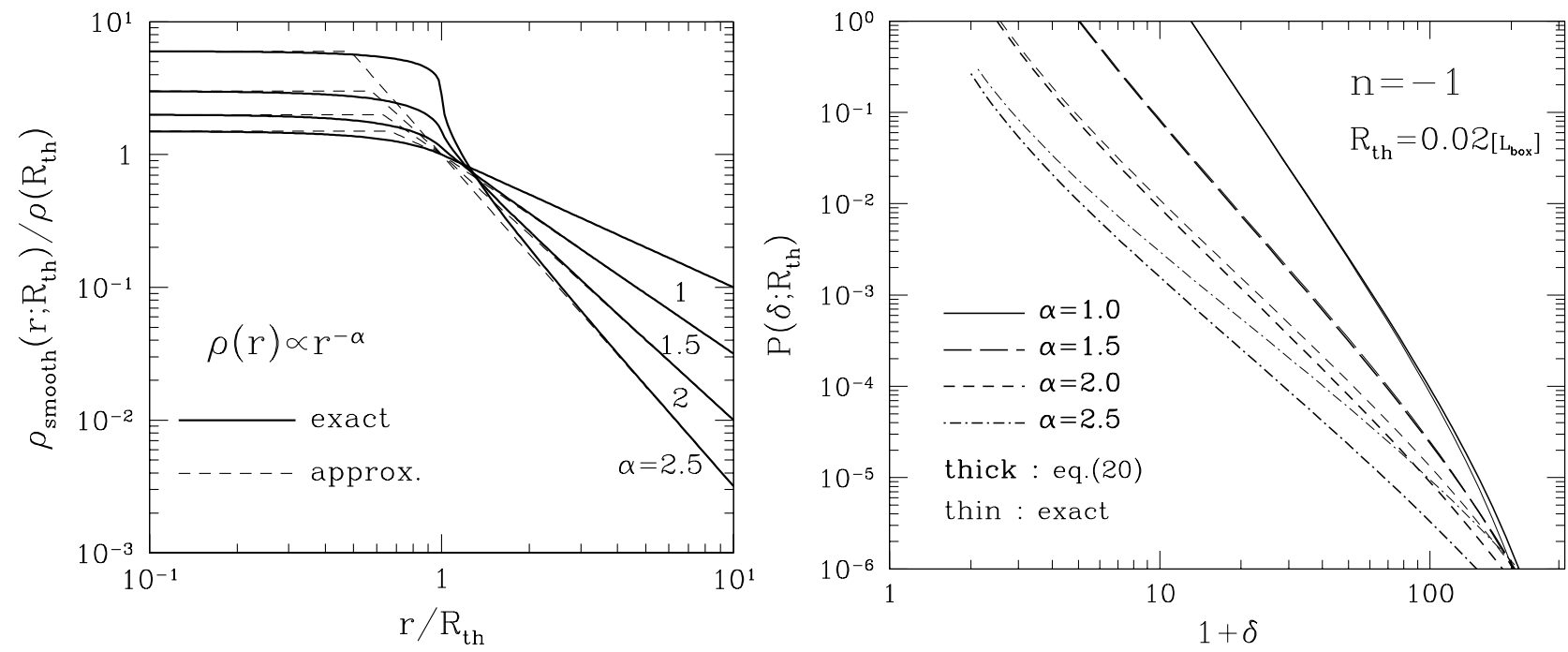

Figure 6. Left panel: comparison of smoothed power-law profiles between exact results (Eqs. 26] 27]; solid) and approximations (Eq: 28 ; dashed). Right panel: the PDFs computed from the exact results without using the approximation (12) (thin lines) compared with the approximation results.

To check the validity of the approximation (20), together with the analytic PDF in the left panel of Figure 2, the PDF computed from the exact results without using approximation (12) is plotted and the results are presented in the right panel of Figure 6 (thin lines). As is expected from the left panel, the approximate expression (20) for steeper halo profiles with $\alpha=2.5$ tends to lower its amplitude, but the qualitative behavior still remains correct.

\section{APPENDIX B: EFFECTS ON UNCERTAINTY OF THE MODEL PREDICTION}

The analytic model of one-point PDF presented in section 2 suffers from uncertainties in the model assumptions (mass function of dark halos and halo density profile). Among these, the concentration parameter of the NFW halo profiles, $c(M)$ might be crucial in comparing the analytic prediction with simulations (Sec.3), since the sensitivity to $c(M)$ has been reported in previous study using dark halo approach (e.g., Seljak 2000). Although we adopt the fitting form of the concentration parameter by Bullock et al. (20001) with a particular set of the parameters $\left(c_{*}, \beta\right)$ (see eq. [3] below), we examine the influence of varying the parameters $\left(c_{*}, \beta\right)$.

Figure 7 shows the dependence of the non-Gaussian tails on the variation in concentration parameter assuming the LCDM universe. Upper(lower) panels represent the results fixing $\beta\left(c_{*}\right)$. Although the influence of the variation $\left(c_{*}, \beta\right)$ turns out to be small, the systematic behavior in the tails of PDF seems to vary in a opposite sense: decreasing the parameter $\beta$ or increasing $c_{*}$ suppresses the non-Gaussian tails.

The small influence on the tails of PDF can be easily deduced from the Fourier transform of the NFW profile (22), in which the concentration parameter appears only through the factor $\delta_{c} r_{s}^{3}$, leading to the logarithmic dependence of $c(M)$. On the other hand, apparently opposite behavior for the variation of $\left(c_{*}, \beta\right)$ is explained as follows. First note that decrease of $\beta$ or increase of $c_{*}$ yields more concentrated density field for halos more massive than the nonlinear mass $M_{*}$. This implies that while the number of halos in which the central density exceeding a specific value of $\delta$ increases, the volume of each density profile exceeding $\delta$ conversely decreases in the massive halos. Since the contribution of massive halo is relatively dominant in the tails of PDF, the latter effect finally wins and accordingly the amplitude of one-point PDF decreases.

Except for the details, the systematic uncertainty of the concentration parameter does not affect the final conclusions in section 4 and the dispersion of 0.2 in $\ln c$ (Jing 2000; Bullock et al. 2001) can be safely neglected in our calculation. Note, however, that in the case of the lensing convergence PDF, the dependence of $\left(c_{*}, \beta\right)$ becomes slightly larger than that in the mass density PDF and the systematic behavior of non-Gaussian tails appears in a opposite sense to $P(\delta)$. These differences simply result from the projection of the three-dimensional density field to the two-dimensional field (e.g., Takada \& Jain 2002).

\section{REFERENCES}

Bartelmann M., Schneider P., 2001, Physics Report, 340, 291

Bernardeau F., 1994a, ApJ, 433, 1

Bernardeau F., 1994b, A\&A, 291, 697

Bernardeau F., Kofman L., 1995, ApJ, 443, 479 


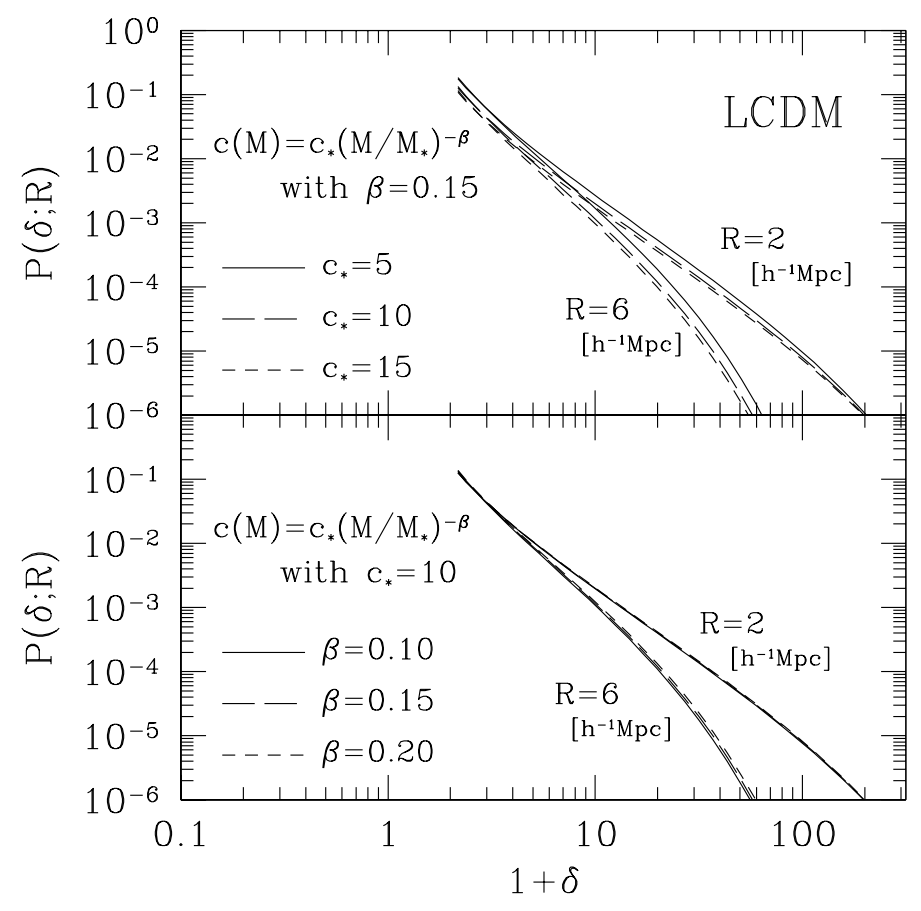

Figure 7. Influence of varying the concentration parameter $c(M)$ on the analytic model of mass density PDFs for $R_{\mathrm{th}}=2 h^{-1} \mathrm{Mpc}$ case and $R_{\mathrm{th}}=6 h^{-1} \mathrm{Mpc}$ case in LCDM model. The upper(lower) panels show the dependence of concentration parameters fixing the $c_{*}(\beta)$.

Bernardeau F., Valageas P., 2000, A\&A, 369, 1

Bouchet F., Strauss M.A., Davis M., Fisher K.B., Yahil A., \& Huchra J.P., 1993, ApJ, 417, 36

Bullock J. S., Kolatt T. S., Sigad Y., Somerville R. S., Kravtsov A. V., Klypin A. A., Primack J. R., Dekel A., 2001, MNRAS, 321, 559

Coles P., Jones B., 1991, MNRAS, 248, 1

Fukushige T., Makino, J., 2001a, ApJ, 557, 533

Fukushige T., Makino, J., 2001b, ApJL, submitted (astro-ph/0108014)

Hamana T., Yoshida N., Suto Y., 2002, ApJ, 568, 455

Hamilton A.J.S., 1985, ApJ, 292, L35

Jing Y. P., 1998, ApJ, 503, L9

Jing Y. P., 2000, ApJ, 535, 30

Jing Y. P., Suto Y., 1998, ApJ, 494, L5

Jing Y. P., Suto Y., 2000, ApJ, 529, L69

Juszkiewicz R., Bouchet, F., Colombi., 1993, ApJ, 419, L9

Kayo I., Taruya A., Suto Y., 2001, ApJ, 561, 22

King I. R., 1962, AJ, 67, 471

Kofman L., Bertschinger E., Gelb J.M. Nusser A., Dekel A,. 1994, ApJ, 420, 44

Kruse G., Schneider, P., 2000, MNRAS, 318, 321

Ma C.-P., Fry J.N., 2000, ApJ, 543, 503

Mellier Y., 1999, ARA\&A, 37, 127

Miyazaki S., Hamana T., Shimasaku K., Furusawa H., Doi M., Hamabe M., Imi K., Kimura M., Komiyama Y., Nakata F., Okada N., Okamura S., Ouchi M., Sekiguchi M., Yagi M., Yasuda N., 2002, ApJL submitted

Moore B., Quinn T., Governato F., Stadel J., Lake G., 1999, MNRAS, 310, 1147

Munshi D., Jain B., 2000, MNRAS, 318, 109

Nakamura T.T., Suto Y., 1997, Prog.Theor.Phys., 97, 49

Navarro J., Frenk C., White S. D. M., 1996, ApJ, 462, 564

Navarro J., Frenk C., White S. D. M., 1997, ApJ, 490, 493

Peacock J.A., Dodds S.J., 1996, MNRAS, 280, L19

Peebles, P.J.E., 1980, The Large-Scale Structure of the Universe (Princeton Univ. Press)

Press W. H., Schechter P., 1974, ApJ, 187, 425

Reblinsky K., Kruse G., jain B., Schneider P., 1999, A\&A 351, 815

Scoccimarro R., Sheth R. K., Hui L., Jain, B., 2001, ApJ, 546, 652

Seljak U., 2000, MNRAS, 318, 203 
Sheth R. K., Tormen G., 1999, MNRAS, 308, 119

Takada M., Jain B., 2002, MNRAS, in press (astro-ph/0205055)

Taruya A., Takada M., Hamana T., Kayo I., Futamase T., 2002, ApJ, 571, 638

Taylor A.N., Watts P.I.R., 2000, MNRAS, 314, 92

Valageas P., 2000, A\&A, 356, 771

Wu X.-P., Hammer F., 1993, MNRAS, 262, 187 